\title{
Locating the CEPs of QCD Phase Transitions via the Dyson-Schwinger Equation Approach of QCD
}

\author{
Yu-xin Liu', Fei Gao \\ Department of Physics and State Key Laboratory of Nuclear Physics and Technology, \\ Peking University, Beijing 100871, China. \\ E-mail: yxliuepku.edu.cn
}

\begin{abstract}
We describe concisely the Dyson-Schwinger (DS) equation approach of QCD and review briefly the progress of the study on the QCD phase transitions with reporting some new results and focussing on the existence and location of the CEP, via the Dyson-Schwinger equation approach of QCD. By calculating and analyzing the chiral susceptibility, the quark number density fluctuation$\mathrm{s}$, and some of the thermal properties in the sophisticated continuum QCD approach, we show that there exist a CEP for the chiral phase transition, and so does for the confinement-deconfinement phase transition. The CEP for the chiral phase transition locates at $\left\{T_{E}, \mu_{E, B}\right\} \cong\{0.90,2.33\} T_{c}^{\chi}$ with $T_{c}^{\chi}=143 \mathrm{MeV}$, and that for the confinement-deconfinement phase transition is approximately at $\left\{T_{E}, \mu_{E, B}\right\} \cong\{0.85,2.50\} T_{c}^{\chi}$. We also show that the existence and the location of the CEPs in theoretical investigations are governed by the (confinement) interaction length scale embodied in the approach and the reason for the fact that different kind approaches give quite distinct locations for the CEP just results from that difference.
\end{abstract}

9th International Workshop on Critical Point and Onset of Deconfinement - CPOD2014,

17-21 November 2014

ZiF (Center of Interdisciplinary Research), University of Bielefeld, Germany

\footnotetext{
${ }^{*}$ Speaker.

$\dagger$ The work was supported by the National Natural Science Foundation of China under Contract Nos. 11435001 and 11175004, and the National Key Basic Research Program of China under Contract No. G2013CB834400.
} 


\section{Introduction}

It is well known that the main objective of the study on QCD phase transitions is to make clear the phase structure of strong interaction matter at finite temperature and/or finite chemical

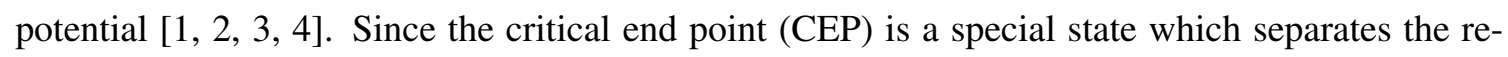
gions of first-order phase transition and crossover (or second-order phase transition), it becomes then the current focus of the theoretical and experimental investigations [ [], [1, [1, 目]. Many criteria, for instance, the disappearance of two minima of the thermodynamical potential, the chiral susceptibility, the disappearance of the S-shape relation between baryon chemical potential (related to the quark chemical potential simply with $\mu_{q}=\mu_{B} / 3$ ) and baryon number density (related to the quark number density simply with $\rho_{q}=3 \rho_{B}$ ), the fluctuations of conserved charges, the finite-size scaling, the thermal conductivity, the quark spectral function, and so on, have been pro-

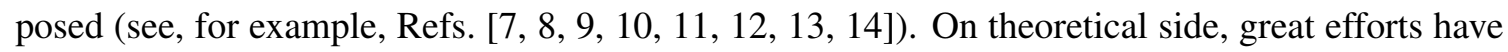
been made to provide information of the phase diagrams, the location of the CEP and the EOS

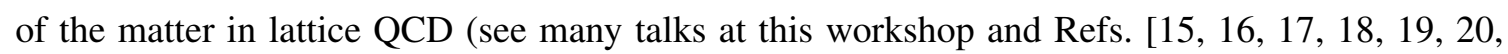

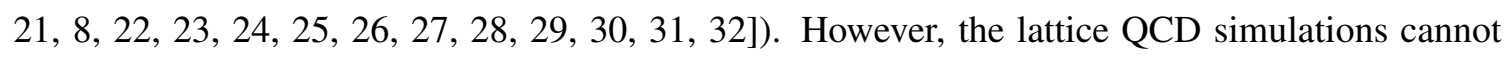
be extended to large chemical potential region at present stage because of the "sign problem". In this case, continuum field theoretical analysis becomes a powerful tool which could work without such a handicap. Plenty of works have then been accomplished in effective models (see, for example, Refs. [33], [34, [35, [36, 137, [38, [39]). Moreover, the Dyson-Schwinger (DS) equations of QCD [40, 4] ] which are believed to be the unique continuous field theory scheme that demonstrates both the dynamical chiral symmetry breaking (DCSB) and the confinement simultaneously [42], have also been implemented to investigate not only the QCD phase transitions including the possibility of the existence and its location of the CEP [43], 44, 45, प, 46, [4, 47, 48, 49] but also the property of the system at the temperature above the pseudo-critical one for the chiral symmetry restoration [50, 5]].

In this talk, we review briefly the progress and report some new results of the study on the QCD phase transitions, with focus on the existence and location of the CEP, via the Dyson-Schwinger equation approach of QCD.

\section{The Approach}

The DS equations of QCD are generally an infinite but countable tower of integral equations coupling the quark, gluon and ghost fields. In practical calculations, one should truncate the series with the scheme preserving the symmetries of QCD. To solve the finite set of the equations concretely, one takes two algorithms at present stage. One is solving the coupled equations with vertex up to three lines (see, for instance, Refs. [46, 52, 53], 54]), another is solving the quark equation with models of the dressed gluon propagator and quark-gluon interaction vertex. We take the later in our calculations.

At finite temperature and quark chemical potential, the quark's gap equation reads

$$
\begin{aligned}
S\left(\vec{p}, \tilde{\omega}_{n}\right)^{-1} & =i \vec{\gamma} \cdot \vec{p}+i \gamma_{4} \tilde{\omega}_{n}+m_{0}+\Sigma\left(\vec{p}, \tilde{\omega}_{n}\right), \\
\Sigma\left(\vec{p}, \tilde{\omega}_{n}\right) & =T \sum_{l=-\infty}^{\infty} \int \frac{d^{3} q}{(2 \pi)^{3}} g^{2} D_{\mu v}\left(\vec{p}-\vec{q}, \Omega_{n l} ; T, \mu\right) \frac{\lambda^{a}}{2} \gamma_{\mu} S\left(\vec{q}, \tilde{\omega}_{l}\right) \frac{\lambda^{a}}{2} \Gamma_{v}\left(\vec{q}, \tilde{\omega}_{l}, \vec{p}, \tilde{\omega}_{n}\right),
\end{aligned}
$$


where $S\left(\vec{p}, \tilde{\omega}_{n}\right)$ is the quark propagator , $m_{0}$ is the current quark mass, $\tilde{\omega}_{n}=\omega_{n}+i \mu$ with $\omega_{n}=(2 n+$ 1) $\pi T$ being the quark Matsubara frequency, and $\mu$ the quark chemical potential $\mu_{q}, \Omega_{n l}=\omega_{n}-\omega_{l}$; $D_{\mu v}$ is the dressed-gluon propagator; and $\Gamma_{v}$ the dressed-quark-gluon vertex.

Considering the Lorentz structure, the quark propagator can be decomposed as

$$
S\left(\vec{p}, \tilde{\omega}_{n}\right)^{-1}=i \vec{\gamma} \cdot \vec{p} A\left(\vec{p}^{2}, \tilde{\omega}_{n}^{2}\right)+i \gamma_{4} \tilde{\omega}_{n} C\left(\vec{p}^{2}, \tilde{\omega}_{n}^{2}\right)+B\left(\vec{p}^{2}, \tilde{\omega}_{n}^{2}\right) .
$$

The dressed-gluon propagator has the form

$$
g^{2} D_{\mu v}\left(\vec{k}, \Omega_{n l}\right)=P_{\mu v}^{T} D_{T}\left(\vec{k}^{2}, \Omega_{n l}^{2}\right)+P_{\mu v}^{L} D_{L}\left(\vec{k}^{2}, \Omega_{n l}^{2}\right)
$$

where $P_{\mu \nu}^{T, L}$ are, respectively, the transverse and longitudinal projection operators, and

$$
D_{T}\left(k_{\Omega}\right)=\mathscr{D}\left(k_{\Omega}^{2}, 0\right), \quad D_{L}\left(k_{\Omega}\right)=\mathscr{D}\left(k_{\Omega}^{2}, m_{g}^{2}\right),
$$

where $m_{g}$ is the thermal mass of the gluon and can be taken as $m_{g}^{2}=16 / 5\left(T^{2}+6 \mu^{2} /\left(5 \pi^{2}\right)\right)$ according to perturbative QCD calculations (see, for example, Ref. [55]).

To solve the equation, one can take the bare vertex approximation or the Ball-Chiu ansatz [56], which includes the restriction of the Ward-Takahashi identity on the longitudinal part, or the anomalous chromomagnetic moment (ACM) model (CLR model) [57], which takes both the longitudinal and the transverse Ward-Takahashi identities into account, for the quark-gluon interaction vertex. For the dressed gluon propagator, one takes commonly the Maris-Tandy (MT) model [58] or the infrared constant model (Qin-Chang, or more briefly, QC, model) [ [5]]. Previous calculations indicate that the general feature of the phase transitions does not depend strongly on the details of the dressed quark-gluon vertex and the dressed gluon propagator (see, e.g., Ref. [5]]). We can then take the approximation $\Gamma_{v}\left(\vec{q}, \tilde{\omega}_{l}, \vec{p}, \tilde{\omega}_{n}\right)=\gamma_{v}$ simply for the quark-gluon vertex and the Qin-Chang gluon model [\$] for the gluon propagator, which reads

$$
\mathscr{D}\left(k_{\Omega}^{2}, m_{g}^{2}\right)=8 \pi^{2} D \frac{1}{\omega^{4}} e^{-s_{\Omega} / \omega^{2}}+\frac{8 \pi^{2} \gamma_{m}}{\ln \left[\tau+\left(1+s_{\Omega} / \Lambda_{\mathrm{QCD}}^{2}\right)^{2}\right]} \mathscr{F}\left(s_{\Omega}\right),
$$

with $\mathscr{F}\left(s_{\Omega}\right)=\left(1-e^{-s_{\Omega} / 4 m_{t}^{2}}\right) / s_{\Omega}, s_{\Omega}=\Omega^{2}+\vec{k}^{2}+m_{g}^{2}, \tau=e^{2}-1, m_{t}=0.5 \mathrm{GeV}, \gamma_{m}=12 / 25$, and $\Lambda_{\mathrm{QCD}}^{N_{f}=4}=0.234 \mathrm{GeV}$. To consider the temperature effect which would screen the interaction, we can remedy the coupling $D$ to $D(T, \mu)$ as

$$
D(T, \mu)= \begin{cases}D, & T<T_{\mathrm{p}}, \\ \frac{a}{b(\mu)+\ln \left[T^{\prime} / \Lambda_{Q C D}\right]}, & T \geq T_{\mathrm{p}}\end{cases}
$$

where $T^{\prime}=\sqrt{T^{2}+6 \mu^{2} /\left(5 \pi^{2}\right)}, T_{\mathrm{p}}$ is the scale which labels the beginning temperature of the thermal screening. At $\mu=0$ we take $T_{\mathrm{p}}=T_{c}$ and $a=0.029, b=0.521$; while for finite chemical potential, the phase transition temperature $T_{c}$ would change, herein we still apply $T_{\mathrm{p}}=T_{c}$ and adjust the value of $b$ at each chemical potential to make the coupling strength $D\left(T_{c}, \mu\right)=D$.

The two parameters $D$ and $\omega$ in the models are chosen as those reproducing the masses and form factors of $\pi, \rho, K, \phi$ and $\omega$ mesons in vacuum. Calculations show that these observables are insensitive to the variation of $\omega \in[0.3,0.5] \mathrm{GeV}$ in the MT model [44] and $\omega \in[0.4,0.6] \mathrm{GeV}$ in the 
QC model [5] as long as $D \omega=$ constant. The renormalization point can be fixed at $\zeta=19 \mathrm{GeV}$ as Refs. [58], and the $m_{0}$ can be taken as $3.4 \mathrm{MeV}$ or $5.5 \mathrm{MeV}$ for $u$ and $d$ quarks.

Practical calculations indicate that, for the quark in chiral limit, the chiral quark condensate becomes nonzero, the quark gets nonzero dynamical mass and the pion appears as massless particle if the interaction strength reaches a critical value, and for the quark in the case beyond chiral limit, there exists both DCSB and explicit chiral symmetry breaking [60, 6], 62]. Analyzing the quark spectral function with the maximum entropy method manifests that the DS equation approach can describe the confinement-deconfinement phase transition very well and the quark spectral function can be an intuitive measure [I4]. These facts provide further evidences for that the DS equation approach is an fundamental and powerful approach for the study of QCD phase transitions.

\section{The Chiral Susceptibility}

The chiral susceptibility $\chi_{c}$ is usually defined as the derivative of the dynamical quark mass or the chiral quark condensate $\langle\bar{q} q\rangle$ with respect to the temperature $T$, i.e., $\chi_{c}=\frac{\partial M}{\partial T} \propto-\frac{\partial\langle\bar{q} q\rangle}{\partial T}$, or to the chemical potential $\mu_{q}$ or the current quark mass $m_{0}$. The condensate can be calculated with

$$
\langle\bar{q} q\rangle_{m_{0}=0}= \begin{cases}-Z_{4} N_{c} N_{f} \int \frac{d^{4} p}{(2 \pi)^{4}} \operatorname{tr}\left[S(p)_{m_{0} \neq 0}\right], & (T=0=\mu), \\ -Z_{4} N_{c} N_{f} T \sum_{n=-\infty}^{+\infty} \int \frac{d^{3} \vec{p}}{(2 \pi)^{3}} \operatorname{tr}\left[S\left(\vec{p}, \omega_{n}, \mu\right)\right], & (T \neq 0 \neq \mu),\end{cases}
$$

with $Z_{4}$ being the renormalization constant. For that beyond the chiral limit, we take the one given in Ref. [63]. Since the quark propagator $S(p)$ is a function of the $A, B$ and $C$ in Eq. (2.3), the $\chi_{c}$ can then be equivalently expressed as $\frac{\partial B}{\partial T}$. In other word, since $M\left(0, \tilde{\omega}_{0}^{2}\right)=B\left(0, \tilde{\omega}_{0}^{2}\right) / C\left(0, \tilde{\omega}_{0}^{2}\right)$, we have $\frac{\partial M}{\partial T}=\frac{\partial}{\partial T}\left(\frac{B}{C}\right)=\frac{1}{C} \frac{\partial B}{\partial T}-\frac{B}{C^{2}} \frac{\partial C}{\partial T}$. Practical calculations show that the $C$ depends on the $T$ (or $\mu$, or $m_{0}$ ) very weakly, we get then $\chi_{c}=\frac{\partial B}{\partial T}$, or $\chi_{c}=\frac{\partial B}{\partial \mu}$, or $\chi_{c}=\frac{\partial B}{\partial m_{0}}$, for the simplicity of calculation.

Because the dynamical quark mass is determined by the quark condensate, the variation behavior of condensate is then usually taken as a measure of chiral phase transition. On the other hand, one takes generally the sign of the second order derivative of the effective thermodynamical potential to identify the stability of a phase. Since the chiral susceptibility is proportional to the inverse of the second order derivative of the effective thermodynamical potential, one can thus take the shift of the sign of the chiral susceptibility as a signature of the phase transition (for multi-flavor system, the chiral susceptibility should be extended to the susceptibility matrix [38]]). Moreover, the chiral susceptibilities of the DCS phase and the DCSB phase diverge or hump at the same point (state) if the evolution between them is a second order phase transition or a crossover but at different locations for a first-order phase transition. Then the point which separates the regions in which the chiral susceptibilities of the two phases diverge at different states or at the same state is just the CEP. It follows that simultaneous analysis of the chiral susceptibilities of the two phases can not only chart the phase diagram but also identify the existence and location of the CEP.

The obtained variation behaviors of the chiral quark condensate and those of the chiral susceptibilities of both the DCSB phase and the dynamical chiral symmetry (DCS) phase with respect to the temperature at zero chemical potential of the system composed of quarks with $m_{0}=3.4 \mathrm{MeV}$, and those with respect to chemical potential at temperature $T=100 \mathrm{MeV}$ are displayed in Fig. س. 


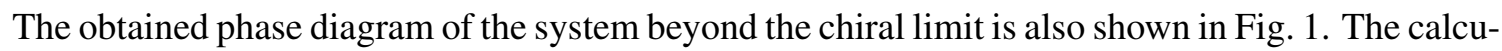
lation indicates that there exists a CEP for the chiral phase transition and it locates at $\left\{T_{E}, \mu_{E, B}\right\} \cong$ $\{0.90,2.33\} T_{c}^{\chi}$ with $T_{c}^{\chi}=143 \mathrm{MeV}$. Such a result is definitely consistent with the lattice QCD results given in Refs. [[], [5, [20] and comparable with those obtained by solving the coupled DS equations [46], but with larger chemical potential than other lattice QCD results [177, 24] and much smaller chemical potential than those in the effective models. One can also observe from the figure that the $\chi_{c}$ beyond the chiral limit varies with temperature in slightly different way from that in chiral limit (as shown in Fig. 1 of Ref. [四]) due to the explicit breaking of the chiral symmetry.
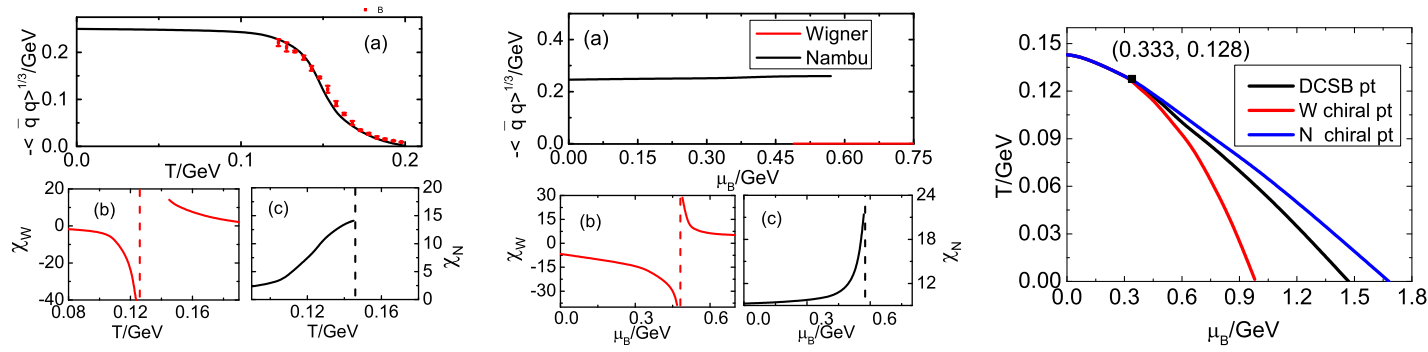

Figure 1: Calculated (in bare vertex and QC model with $D=1.0 \mathrm{GeV}^{2}, \omega=0.5 \mathrm{GeV}$ ) temperature dependence of the quark condensate and comparison with lattice QCD result [B]] (left panel (a)) and the chiral susceptibilities of the DCS phase (left panel (b)) and the DCSB phase (left panel (c)) at zero chemical potential, those of the quark chemical potential dependence at temperature $T=100 \mathrm{MeV}$ (middle panels) and the phase diagram (right panel).

Furthermore, since the chiral susceptibility can be determined directly with the solutions of the DS equation, such a chiral susceptibility criterion can be not only available for the case of which we can have the effective thermodynamical potential but also, especially, powerful for the case where the effective thermodynamical potential is not available when considering the complexity of the non-perturbative nature. An example has been given in the Fig. 3 of Ref. [प].

\section{The Quark Number Density Fluctuations}

It is well known that, for a grand ensemble composed of particles whose chemical potential is $\mu_{X}$, the expectation value of the total particle number $N_{X}$ can be obtained from the grand thermodynamical potential $\Omega\left[\mu_{X} ; T\right]$ with relation

$$
-\frac{\delta \Omega\left[\mu_{X} ; T\right]}{\delta \mu_{X}}=\int d^{4} x\langle\hat{n}(x)\rangle=\overline{N_{X}},
$$

where $\langle\cdots\rangle$ and $\cdots$ denote the ensemble averages. With some derivations, one can have the second order fluctuation $\chi_{2}$ as

$$
\chi_{2}^{X}=\frac{1}{V T^{3}} \overline{\delta N_{X}^{2}}=\frac{1}{V T^{3}} \overline{\left(N_{X}-\overline{N_{X}}\right)^{2}}=\frac{1}{V T^{2}} \frac{\partial \overline{N_{X}}}{\partial \mu_{X}} .
$$

Similarly, one could obtain the higher order fluctuations as

$$
\chi_{3}^{X}=\frac{1}{V T^{3}} \overline{\delta N_{X}^{3}}
$$




$$
\chi_{4}^{X}=\frac{1}{V T^{3}}\left(\overline{\delta N_{X}^{4}}-3{\overline{\delta N_{X}^{2}}}^{2}\right) .
$$

Supposing that the system is uniform, i.e., $\bar{N}=V n$ with $n$ being the particle number density, the fluctuations could be derived from the certain order derivatives of $n(\mu, T)$, which can be obtained directly from the quark propagator with

$$
\begin{aligned}
n(\mu, T) & =2 N_{c} N_{f} Z_{2} \int_{-\infty}^{\infty} \frac{d^{3} \vec{p}}{(2 \pi)^{3}} f_{1}(\vec{p} ; \mu, T), \\
f_{1}(\vec{p} ; \mu, T) & =\frac{T}{2} \sum_{m=-\infty}^{\infty} \operatorname{tr}_{\mathrm{D}}\left(-\gamma_{4} S\left(\tilde{\omega}_{m}, \vec{p}\right)\right),
\end{aligned}
$$

where $Z_{2}$ is the quark wave-function normalization constant, $N_{c}$ is the color number, $N_{f}$ is the flavor number, $S\left(\tilde{\omega}_{m}, \vec{p}\right)$ is the quark propagator, and the summation is taken over the Matsubara frequencies $\left(\tilde{\omega}_{m}=\omega_{m}+i \mu=(2 m+1) \pi T+i \mu, m \in \mathbb{Z}\right)$.

It is apparent that the fluctuations relate directly to the confinement-deconfinement phase transition since they measure the quark number density, and bridge theoretical predictions and experimental data since they influence the hadron yields after the phase transitions and can be measured after the chemical-freeze out.

We have calculated the fluctuations up to the fourth order at not only finite temperature but also finite chemical potential of the system composed of light flavor quarks with $m_{0}=5.5 \mathrm{MeV}$. The obtained results of the variation behaviors with respect to the temperature agree with lattice QCD simulation results very well, as shown in the Figs. 1 and 2 of Ref. [49]. The obtained result of the quark chemical potential dependence of the ratios $\frac{\chi_{3}}{\chi_{2}}$ and $\frac{\chi_{4}}{\chi_{2}}$ at several values of temperature is redisplayed in Fig. 1 . The calculated results show that the variation features of the fluctuations and their ratios are quite similar to those of the chiral susceptibility. They can then be taken to establish the phase diagram and explore the existence of the CEP. The results also indicate that there exists a CEP for the confinement-deconfinement phase transition and it locates at $\left\{T_{E}, \mu_{E, B}\right\} \cong\{0.85,2.46\} T_{c}^{\chi}$ with $T_{c}^{\chi}=146 \mathrm{MeV}$ in the MT model of the dressed gluon propagator and $\left\{T_{E}, \mu_{E, B}\right\} \cong\{0.86,2.49\} T_{c}^{\chi}$ with $T_{c}^{\chi}=150 \mathrm{MeV}$ in the QC model.

Looking over the models of the dressed gluon propagator, one can notice that it involves two parameters $D$ and $\omega$. With the constraint $D \omega=$ const, the two models still have a free parameter $\omega$. Since the $\omega$ characterizes the interaction width in the momentum space. One can then introduce a confinement length scale in the coordinate space as $r_{\omega}=1 / \omega$. If $\omega \rightarrow 0$, we have $r_{\omega} \rightarrow \infty$, i.e., the model is actually the constant interaction model. If $\omega \rightarrow \infty$, we have $r_{\omega} \rightarrow 0$ and the model is in fact the contact interaction model, i.e., the NJL-type model. With nonzero finite values of $\omega$, the model is the interpolation between the two limits. Then, carrying out the calculations with various values of $\omega$, one can investigate the model dependence of the CEP. Our calculated results listed in the Table of Ref. [四] and that of Ref. [4Q] show that the ratio of the $\frac{\mu_{E, q}}{T_{E}}$ (i.e., $\frac{\mu_{E, B}}{T_{E}}$ ) decreases with the increasing of the $r_{\omega}$, and all the values are in the between of the result in the constant interaction model $\left(\frac{\mu_{E, B}}{T_{E}}=0\right.$ ) [43] and that in the contact interaction model (many NJL-like models give $\left.\frac{\mu_{E, B}}{T_{E}}>9\right)$. The underlying mechanism of such a variation feature can be attributed to that the decreasing of $\omega$, or the the enlarging of the confinement length scale, means the enhancing of the binding energy of baryons (more generally, hadrons). It takes then more energy to release quarks from the color singlet system (DCSB phase or DCSB/DCS coexistence phase), so that the 


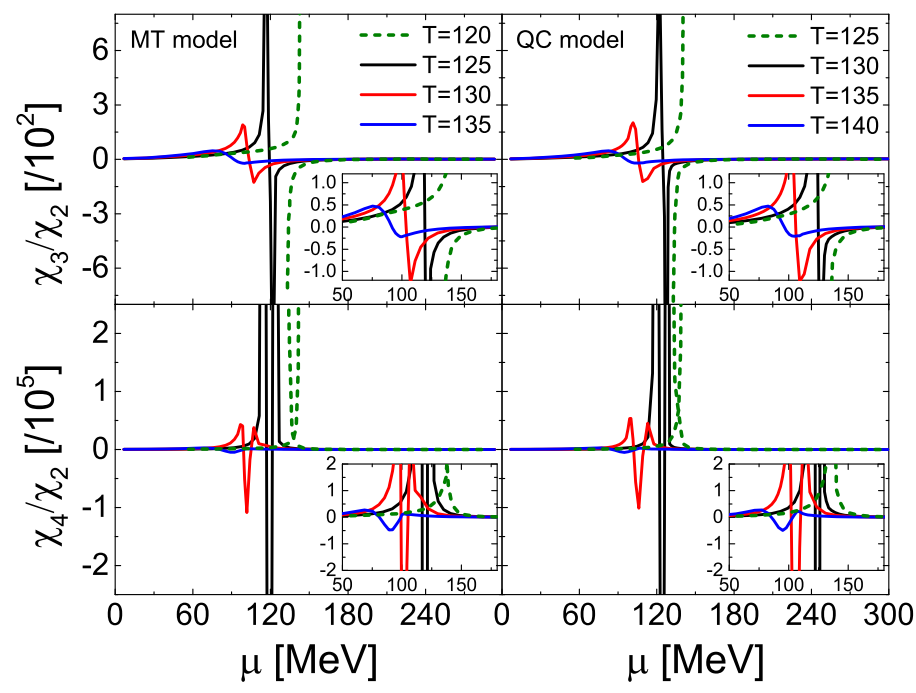

Figure 2: (color online) The quark chemical potential dependence of the ratio of the third to second order fluctuations at several temperatures (upper panels) and that of the fourth to second order fluctuations (lower panels). The left panels show the results with the bare vertex and the MT model with $\omega^{\mathrm{MT}}=0.4 \mathrm{GeV}$, and the right panels in the bare vertex and the QC model with $\omega^{\mathrm{QC}}=0.5 \mathrm{GeV}$.

$T_{E}$ and the $T_{c}$ get higher. Meanwhile, increasing the confinement length scale plays the same role as increasing the density of the system, it compensates then the effect of increasing the chemical potential. Therefore, $\mu_{E}^{q}$ decreases due to the compensation. As a consequence, the $\frac{\mu_{E, B}}{T_{E}}$ decreases with the increasing of the confinement interaction length scale $r_{\omega}$ (i.e., the decreasing of the $\omega$ ). In addition, our results indicate that the fact that different methods give distinct locations of the CEP results from that they take different (confinement) interaction length scales.

\section{The Specific Heat Capability}

The specific heat capability density $c_{V}$ of a system can be fixed with

$$
c_{V}=\frac{\partial \varepsilon}{\partial T}=\frac{\partial\left(T^{2} \frac{\partial(p / T)}{\partial T}\right)}{\partial T},
$$

where $p$ is the pressure of the system, which can be given as

$$
p[S]=\frac{T}{V} \ln Z=\frac{T}{V}\left(\operatorname{Tr} \ln \left[\beta S^{-1}\right]-\frac{1}{2} \operatorname{Tr}[\Sigma S]\right),
$$

with $\beta=1 / T, \Sigma=S^{-1}-S_{0}^{-1}$ and $S_{0}$ the free quark propagator.

With sophisticated subtraction scheme, one can remove the ultraviolet divergence and accomplish the calculation. The obtained result of the temperature dependence of the specific heat capability density at several values of the baryon chemical potential is illustrated in Fig. [1].

The figure manifests evidently that the $c_{V}$ varies with respect to the temperature and the chemical potential continuously in the low chemical potential and high temperature region and it involves a discontinuity in the intermediate chemical potential and low temperature region after a 


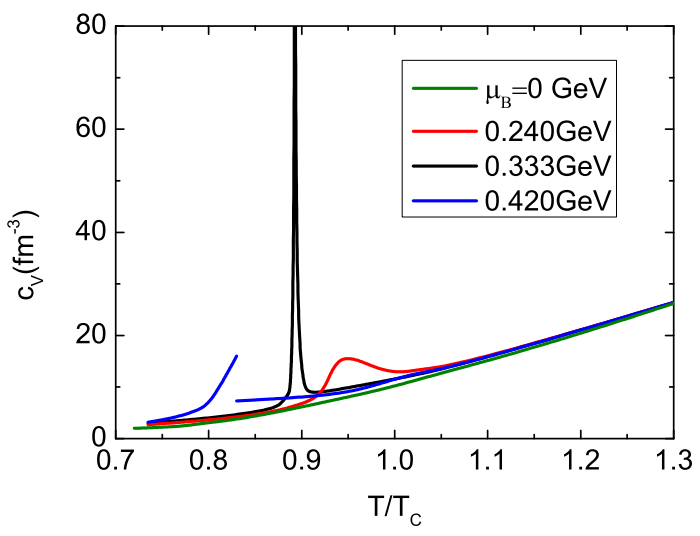

Figure 3: Calculated temperature dependence of the specific heat capability density at several baryon chemical potentials (for the matter composed of two light flavor quarks beyond chiral limit with $m_{0}=3.4 \mathrm{MeV}$ ).

divergence. According to the general principle of thermodynamics, such a feature is an apparent demonstration for that the phase transition in low chemical potential and high temperature region is in fact a crossover, that in intermediate (high) chemical potential and low temperature region is really a first-order phase transition, and the state (point) for the $c_{V}$ to diverge is just the CEP. We show further there exists a CEP and it locates at $\left\{T_{E}, \mu_{E, B}\right\} \cong\{0.90,2.33\} T_{c}^{\chi}$ with $T_{c}^{\chi}=143 \mathrm{MeV}$, which is exactly the same as that given by analyzing the chiral susceptibility.

\section{Summary and Remarks}

We have reviewed briefly the progress and reported some new results of the study on the QCD phase transitions, with focus on the existence and location of the CEP, via the Dyson-Schwinger equation approach of QCD. By calculating and analyzing the chiral susceptibility, the quark number density fluctuations, and some of the thermal properties in the sophisticated continuum QCD approach, we show that there exist a CEP for the chiral phase transition, and so does for the confinement-deconfinement phase transition. The CEP for the chiral phase transition locates at $\left\{T_{E}, \mu_{E, B}\right\}=\{0.90,2.33\} T_{c}^{\chi}$ with $T_{c}^{\chi}=143 \mathrm{MeV}$, and that for the confinement-deconfinement phase transition is approximately at $\left\{T_{E}, \mu_{E, B}\right\}=\{0.85,2.50\} T_{c}^{\chi}$. We also show that the existence and the location of the CEPs in theoretical investigations are governed by the (confinement) interaction length scale embodied in the approach. In addition, the reason for the fact that different kind approaches give quite distinct locations for the CEP is given.

Recalling the algorithm for the calculations in the DS equation scheme, one has not yet taken into account the temperature and the chemical potential effects in the fundamental view of QCD. In order to get more solid result via the DS equation approach, it needs then great efforts for us to devote to such an aspect. In addition, inferring the result for hadron observables in Ref. [64], we should pay much more attention to the quark-gluon interaction vertex so that it can include not only the restriction of the Ward-Takahashi identity for the longitudinal part but also that for the transverse part appropriately, for instance, carrying out calculations with the ACM model. 


\section{References}

[1] P. Braun-Munzinger, and J. Wambach, Rev. Mod. Phys. 81, 1031 (2009).

[2] S. Gupta, X. Luo, B. Mohanty, H. G. Ritter, and N. Xu, Science 332, 1525 (2011).

[3] K. Fukushima, and T. Hatsuda, Rep. Prog. Phys. 74, 014001 (2011).

[4] O. Philipsen, Prog. Part. Nucl. Phys. 70, 55 (2013).

[5] G. L. Melkumov, et al., Nucl. Phys. B (Proc. Suppl.) 219, 102 (2011); G. L. Melkumov, et al.(NA49 Collaboration), Phys. At. Nucl. 75, 556 (2012).

[6] G. Odyniec (for the STAR collaboration), Phys. At. Nucl. 75, 602 (2012).

[7] S. X. Qin, L. Chang, H. Chen, Y. X. Liu and C. D. Roberts, Phys. Rev. Lett. 106, 172301 (2011).

[8] A. Y. Li, A. Alexandru, K.-F. Liu, and X. Meng, Phys. Rev. D 82, 054502 (2010); A. Y. Li, A. Alexandru, and K.-F. Liu, Phys. Rev. D 84, 071503(R) (2011).

[9] M. A. Stephanov, K. Rajagopal, and E.V. Shuryak, Phys. Rev. Lett. 81, 4816 (1998); M. A. Stephanov, Phys. Rev. Lett. 102, 032301 (2009).

[10] M. A. Stephanov, Phys. Rev. Lett. 107, 052301 (2011).

[11] M. Asakawa, S. Ejiri, and M. Kitazawa, Phys. Rev. Lett. 103, 262301 (2009).

[12] L. F. Palhares, E. S. Fraga, and T. Kodama, J. Phys. G 37, 094031 (2010); J. Phys. G 38, 085101 (2011); E. S. Fraga, L. F. Palhares, and P. Sorensen, Phys. Rev. C 84, 011903(R) (2011).

[13] J. I. Kapusta, and J. M. Torres-Rincon, Phys. Rev. C 86, 054911 (2012).

[14] S. X. Qin, and D. H. Rischke, Phys. Rev. D 88, 056007 (2013).

[15] Z. Fodor, and S. D. Katz, J. High Energy Phys. 03 (2002), 014. J. High Energy Phys. 04 (2004), 050.

[16] P. de Forcrand, O. Philipsen, Nucl. Phys. B 642, 290 (2002); Nucl. Phys. B 673, 170 (2003).

[17] R. V. Gavai and S. Gupta, Phys. Rev. D 71, 114014 (2005); Phys. Rev. D 78, 114503 (2008),

[18] H. S. Chen, and X. Q. Luo, Phys. Rev. D 72, 034504 (2005).

[19] S. Ejiri, Phys. Rev. D 77, 014508 (2008).

[20] C. Schmidt (for the RBC-Bielefeld Collaboration), J. Phys. G 35, 104093 (2008).

[21] G. Endrödi, Z. Fodor, S.D. Katz, and K. Szabó, J. High Energy Phys. 04 (2011), 001.

[22] O. Kaczmarek, et al., Phys. Rev. D 83, 014504 (2011).

[23] P. Cea, L. Cosmai, and A. Papa, Phys. Rev. D 89, 074512 (2014).

[24] S. Gupta, N. Karthik, and P. Majumdar, Phys. Rev. D 90, 034001 (2014).

[25] Z. Fodor, S.D. Katz, K.K. Szabó, Phys. Lett. B 568, 73(2003).

[26] C.R. Allton, et al., Phys. Rev. D 68, 014507 (2003).

[27] C. DeTar, et al., Phys. Rev. D 81, 114504 (2010).

[28] M. Cheng, et al, Phys. Rev. D 77, 014511 (2008).

[29] A. Bazavov, T. Bhattacharya, M. Cheng, et al, Phys. Rev. D 80, 014504 (2009).

[30] P. Huovinen, and P. Petreczky, Nucl. Phys. A 837, 26 (2010).

[31] Sz. Borsányi, et al., J. High Energy Phys. 1009, 073 (2010).

[32] Sz. Borsányi, et al., J. High Energy Phys. 1208, 053 (2012).

[33] B. J. Schaefer, J. M. Pawlowski, and J. Wambach, Phys. Rev. D 76, 074023 (2007).

[34] W. J. Fu, Z. Zhang, and Y. X. Liu, Phys. Rev. D 77, 014006 (2008).

[35] M. Ciminale, R. Gatto, N. D. Ippolito, G. Nardulli, and M. Ruggieri, Phys. Rev. D 77, 054023 (2008). 
[36] K. Fukushima, Phys. Rev. D 77, 114028 (2008).

[37] Y. Sakai, T. Sasaki, H. Kouno, and M. Yahiro, Phys. Rev. D 82, 076003 (2010).

[38] L. J. Jiang, X. Y. Xin, K. L. Wang, S. X. Qin, and Y. X. Liu, Phys. Rev. D 88, 016008 (2013).

[39] X. Y. Xin, S. X. Qin, and Y. X. Liu, Phys. Rev. D 89, 094012 (2014).

[40] C. D. Roberts, and A. G. Williams, Prog. Part. Nucl. Phys. 33, 477 (1994); C. D. Roberts and S. M. Schmidt, Prog. Part. Nucl. Phys. 45, S1 (2000); P. Maris and C. D. Roberts, Int. J. Mod. Phys. E 12, 297 (2003). A. Bashir, L. Chang, I. C. Cloet, B. El-Bennich, Y. X. Liu, C. D. Roberts, and P. C. Tandy, Commun. Theor. Phys. 58, 79 (2012); I.C. Cloet, and C. D. Roberts, Prog. Nucl. Part. Phys. 77, 1 (2014)

[41] R. Alkofer, and L. von Smekal, Phys. Rept. 353, 281 (2001); C. S. Fischer, J. Phys. G 32, R253 (2006).

[42] L. McLerran and R. D. Pisarski, Nucl. Phys. A 796, 83 (2007).

[43] D. Blaschke, C. D. Robers, S. Schmidt, Phys. Lett. B 425, 232 (1998).

[44] P. Maris, A. Raya, C. D. Roberts, and S. M. Schmidt, Eur. Phys. J. A 18, 231 (2003).

[45] C. S. Fischer, Phys. Rev. Lett. 103, 052003 (2009); C. S. Fischer, and J. A. Mueller, Phys. Rev. D 80, 074029 (2009).

[46] C. S. Fischer, J. Luecker, and J. A. Mueller Phys. Lett. B 702, 438 (2011); C. S. Fischer, J. Luecker, Phys. Lett. B 718, 1036 (2013); C. S. Fischer, J. Luecker, and C. A. Welzbacher, Phys. Rev. D 90, 034022 (2014).

[47] E. Gutiérrez, A. Ahmad, A. Ayala, A. Bashir, and A. Raya, Phys. Lett. B 718, 1036 (2013).

[48] M. He, J. F. Li, W. M. Sun, and H. S. Zong, Phys. Rev. D 79, 036001 (2009); B. Wang, Z. F. Cui, W. M. Sun, and H. S. Zong, Few-Body Syst. 55, 47 (2014); C. Shi, Y. L. Wang, Y. Jiang, Z. F. Cui, and H. S. Zong, J. High Energy Phys. 07 (2014), 014 (2014).

[49] X.Y. Xin, S.X. Qin, and Y.X. Liu, Phys. Rev. D 90, 076006 (2014).

[50] J. A. Mueller, C. S. Fischer, and D. Nickel, Eur. Phys. J. C 70, 1037 (2010); S. X. Qin, L. Chang, Y. X. Liu, and C. D. Roberts, Phys. Rev. D 84, 014017 (2011).

[51] F. Gao, S. X. Qin, Y. X. Liu, C. D. Roberts, and S. M. Schmidt, Phys. Rev. D 89, 076009 (2014).

[52] L. von Smekal, A. Hauck, and R. Alkofer, Ann. Phys. 267, 1 (1998).

[53] A. C. Aguilar, D. Binosi, and J. Papavassiliou, Phys. Rev. D 86, 014032 (2012).

[54] M. Hopfer, R. Alkofer, and G. Haase, Compt. Phys. Commun. 184, 1183 (2013).

[55] N. Haque, M. G. Mustafa, and M. Strickland, Phys. Rev. D 87, 105007 (2013).

[56] J. S. Ball and T. -W. Chiu, Phys. Rev. D 22, 2542 (1980).

[57] L. Chang, Y. X. Liu, and C. D. Roberts, Phys. Rev. Lett. 106, 072001 (2011). S. X. Qin, L. Chang, Y. X. Liu, C. D. Roberts and S. M. Schmidt, Phys. Lett. B 722, 384 (2013);

[58] P. Maris and P. C. Tandy, Phys. Rev. C 60, 055214 (1999).

[59] S. X. Qin, L. Chang, Y. X. Liu, C. D. Roberts, and D. J. Wilson, Phys. Rev. C 84, 042202(R) (2011).

[60] C. D. Roberts, B. H. J. McKellar, Phys. Rev. D 41 , 672 (1990).

[61] L. Chang, et al., Phys. Rev. C 75, 015201 (2007); K. L. Wang, et al., Phys. Rev. D 86, 114001 (2012).

[62] R. Williams, C. S. Fischer, and M. R. Pennington, Phys. Lett. B 645, 167 (2007); C. S. Fischer, D. Nickel, and R. Williams, Eur. Phys. J. C 60, 47 (2009).

[63] A. Bender, D. Blaschke, Y. Kalinovsky, and C. D. Roberts, Phys. Rev. Lett. 77, 3724 (1996).

[64] D. Binosi, L. Chang, J. Papavassiliou, and C. D. Roberts, Phys. Lett. B 742, 183 (2015). 